\title{
Monitoramento de Aedes spp. Com Armadilhas Ovitrampa Instaladas em Diferentes Posições
}

\author{
Monitoring of Aedes spp. with Ovitrap Traps Installed in Different Positions
}

\author{
Leidiane da Silva Barreto de Oliveira*a; Franciele Rodrigues de Lima ${ }^{\mathrm{a}}$; Marcelo Dias de Souza ; Alexandre Roman \\ Parada $^{\text {b; Wanderson Batista da Silva }}{ }^{\mathrm{b}}$
}

${ }^{a}$ Universidade de Cuiabá. MT, Brasil.

'Universidade de Cuiabá, Programa de Pós-Graduação Strcito Sensu em Ciências Ambientais. MT, Brasil.

*E-mail: leidykerllott@hotmail.com

\begin{abstract}
Resumo
Um fator que ocorre em centros urbanos é a proliferação de mosquitos vetores de doenças, tais como o Aedes aegypti, sendo que em muitos casos os criadouros de mosquito ocorrem devido ao crescimento desorganizado das cidades que propiciam ambientes adequados para a proliferação desses vetores. Este estudo teve como objetivo analisar o monitoramento de mosquitos Aedes spp. em área nativa no município de Cuiabá, MT, bem como, comparar o efeito da atratividade das armadilhas ovitrampas com diferentes coloração e posição de instalação. Na área de estudo foram instaladas armadilhas em quatro pontos de amostragem, sendo que cada ponto continha 4 armadilhas: duas instaladas na posição de um metro acima do solo e outras duas instaladas ao nível do solo, sendo uma de coloração branca e outra de coloração preta em cada ponto, totalizando 16 armadilhas no ambiente. O delineamento estatístico utilizado foi um fatorial $2 \times 2$ sendo o primeiro fator a posição e o segundo fator a cor da armadilha. As populações dos mosquitos foram estimadas a partir de larvas e ovos coletados nas armadilhas ovitrampas, em quatro coletas semanais durante 42 dias. Houve diferença significativa entre as colorações das armadilhas e posição de instalação, demostrando que os mosquitos Aedes aegypti preferem a armadilha de cor preta a $1 \mathrm{~m}$ de altura. Já para os mosquitos da espécie Aedes albopictus houve diferença significativa apenas entre a coloração das armadinhas, evidenciando que este mosquito prefere as armadilhas de cor preta.
\end{abstract}

Palavras-chave: Aedes aegypti. Aedes albopictus. Diversidade. Vetores.

\begin{abstract}
The objective of this study was analyze the monitoring of Aedes spp. mosquitoes in a native area in the city of Cuiabá, MT, as well as to compare the effect of the attractiveness of ovitraps traps with different color and installation. In the study area, traps were installed in four sampling points, being each point containing 4 traps: two installed at a position of one meter above the ground and two others installed at ground level, being one white and the other black in each point, totaling 16 traps in the environment. The statistical design used was a factorial $2 \times 2$, being the first factor the position and the second factor the color of trap. The mosquitoes populations were estimated from larvae and eggs collected in ovitraps traps, in four collections weekly in 42 days. There was a significant difference between the staining of the traps and installation position, demonstrating that Aedes aegypti mosquitoes prefer the black trap and in aerial position. However, for mosquitoes of the Aedes albopictus species, there was a statistical difference between the coloring of the enclosures, evidencing that this specie prefer the black traps.
\end{abstract}

Keywords: Aedes aegypti. Aedes albopictus. Diversity. Vectors.

\section{Introdução}

As espécies Aedes aegypti e Aedes albopictus, pertencem a ordem díptera, família Culicidae, e são de grande relevância para a saúde pública, pois são transmissores de diversas arboviroses prejudiciais à saúde humana e dos animais (MILLER; BALLINGER, 1988). O Ae. aegypti, é o vetor de varias doenças virais, em especial as ocasionadas pelo vírus do gênero Flavivírus, conhecido como dengue, sendo autor da transmissão em epidemias registradas desde o início dos anos 1980 (CONSOLI; OLIVEIRA, 1994). O Ae albopictus, ainda não foi identificado como vetor natural do vírus dengue no Brasil (DEGALLIER et al., 2003), apesar de já ter sido atestado que, em condições de laboratório, as populações brasileiras desta espécie têm a habilidade de se contaminar com o vírus e transmiti-lo (MILLER; BALLINGER, 1988; OLIVEIRA et al., 2003; CASTRO et al., 2004).

O Ae. aegypti é originário da África, vive em áreas tropicais e subtropicais, embora seja encontrado em todos os continentes, localiza-se preferencialmente na zona urbana, e dentro ou nos arredores dos imóveis. Essa espécie realiza a oviposição principalmente em reservatórios artificiais com água parada, como pneus, garrafas, latas, potes, vasos e pratos de planta, piscinas sem tratamento e reservatórios de água destampados (TAUIL, 2002).

$\mathrm{O}$ agrupamento populacional decorrido da urbanização traz algumas consequências, como aumento de resíduos em locais inapropriados, da qual podem proporcionar recipientes artificiais, que são elementos determinantes no desenvolvimento do Ae. aegypti nos centros urbanos das regiões tropicais e subtropicais do planeta. A disponibilidade de um local para oviposição é um dos principais fatores encarregados pela distribuição dos mosquitos nos criadouros e sua consecutiva propagação em diferentes áreas geográficas (TILAK et al., 2004).

De acordo com Santos (2008), más condições 
socioambientais, resultantes da urbanização acelerada, promovem o rápido desenvolvimento do Ae. aegypti, devido o aumento de resíduos urbanos dispostos irregularmente. As recentes modificações climáticas, como o aquecimento global, e a intensidade de circulação internacional de pessoas e produtos também contribuem para sua proliferação.

$O$ Ae. aegypti se alimenta de seivas das plantas. No entanto, as fêmeas desta espécie são hematófagas, e também se alimentam de sangue. A alimentar-se de sangue de um hospedeiro contaminado, ela pode inserir junto, o microorganismo causador da doença, no caso a dengue (SILVA et al., 2008).

O Ae. aegyptis hospeda-se geralmente dentro das casas, devido a inúmeros abrigos que esses ambientes possuem. Machos e fêmeas podem ser coletados em cantos escuros das casas, em baixo de camas, atrás de armários, sob pias de cozinhas, no interior de banheiros, entre outros possíveis abrigos. São encontrados em maior quantidade nos quintais ou áreas abertas (BARATA et al., 2001).

Diferente do Ae. aegyptis, o Ae. albopictus se estende nos ambientes urbano, suburbano e rural, em regiões com menor concentração de pessoas (CONSOLI, OLIVEIRA, 1994). Esse inseto também é adaptado ao ambiente domiciliar tendo como criadouros recipientes de utilização doméstica como jarros, tambores, pneus e tanques. No meio rural está presente em nichos naturais como ocos de árvores, em amontoados de folhas e em orifícios de bambus. A fêmea do mosquito tem como fonte de alimento o sangue humano, e de outros mamíferos ou até mesmo aves (BRASIL, 2001, p.18).

Apesar de o Ae. albopictus também ser visto em vasilhames temporários, essa espécie prefere o habitat natural da floresta. Em ambientes urbanos desenvolve mais frequentemente, fora das casas, em jardins; e com menor frequência dentro destas, em vasilhames artificiais (BRAGA; VALLE, 2007).

As formas imaturas e adultas do Ae. albopictus, são bastante semelhantes às do Ae. aegypti em muitos aspectos morfológicos como locais apropriados para o desenvolvimento e ciclo de vida, pois ambas as espécies, apresenta quatro fases: ovo, larva, pupa e adulto. A duração dos ciclos de vida a partir da oviposição até a fase adulta é em torno de 10 dias, principalmente, de temperatura e material orgânico para alimentação em condições propicias, sendo que o adulto pode sobreviver por mais 30 dias. O Ae. aegypti este está entre as espécies de mosquito que passa mais rapidamente pela fase imatura (estádios de ovo, larva e pupa), por isso são capazes de multiplicarem-se em recipientes pequenos com água, antes que sequem (OLIVEIRA, 2013).

Apesar de existir muitas semelhanças entre o comportamento do Ae. aegypti e do Ae. albopictus, as diferenças entre eles são categóricas para a dinâmica de transmissão das doenças, proliferação dos vírus e dispersão da espécie, pois Ae. albopictus são encontrados mais frequentemente em ambientes naturais, enquanto Ae. aegypti em ambientes urbanos (FORATTINI 2002). As condições climáticas como, temperatura, pluviosidade e altitude, intervêm no ciclo vital do Ae. aegypti, além da domiciliação, disseminação, alimentação e reprodução (DONALÍSIO; GLASSER, 2002).

As atividades humanas têm um papel dominante na distribuição dos vetores. O fluxo populacional influencia diretamente a difusão do vírus que os vetores são capazes de transmitir, e em cidades de grande porte a ocupação irregular do espaço, forma paisagens que podem causar estratos distintos de transmissão de dengue, consideravelmente no que se refere à permanência de habitats favoráveis ao vetor (COSTA; NATAL, 1998).

Como grande parte da população não se preocupa em destinar os resíduos corretamente, principalmente sólidos, e ainda tampar caixas d'água, retirar qualquer objeto que possa acumular á mesma, o mosquito encontra condições favoráveis para a proliferação (LUCIANO-ALMEIDA; KOETZ, 2011). Perante aos desafios do controle dos vetores e de um quadro grave e preocupante com relação a ocorrência de arboviroses apresentadas pela disseminação destes vírus em todo o mundo, torna-se indispensável à adoção de táticas específicas, em especial, metodologias adequadas, que permitam o correto monitoramento dos vetores que possam nortear políticas públicas e redes de vigilância e combate a essas doenças (ZARA et al., 2016).

O uso de armadilhas para monitoramento destes vetores é recomendado para avaliar a ocorrências destas espécies em áreas de risco, geralmente associada aos licenciamentos ambientais (ARAÚJO, 2019). Estudos indicam a instalação das armadilhas sempre em coloração escura e instaladas em altura superior a $1 \mathrm{~m}$ para monitoramento de mosquito (SOUZA, 2010).

Desse modo este estudo tem como objetivo analisar a eficiência no monitoramento de Aedes spp. utilizando armadilhas tipo ovitrampas instaladas em área nativa no município de Cuiabá, MT, comparando o efeito da atratividade da coloração e posição das armadilhas.

\section{Material e Métodos}

\subsection{Localização e descrição da Área}

O estudo foi desenvolvido no município de CuiabáMT, cujo o clima dominante é do tipo tropical continental semi-úmidode acordo com a classificação de Köppen, com temperatura média anual em torno de $25^{\circ}$ a $26^{\circ} \mathrm{C}$ e índice pluviométrico anual entre 1.250 a $1.500 \mathrm{~mm}$ (ALMEIDA et al., 2011).

A área de estudo localiza-se nas coordenadas geográficas $15^{\circ} 36^{\prime} 45^{\prime \prime} \mathrm{S}$ e $56^{\circ} 6^{\prime} 46^{\prime \prime} \mathrm{W}$, cota altimétrica de $160 \mathrm{~m}$ em relação ao nível do mar, e área de aproximadamente 34.245,21 $\mathrm{m}^{2}$, com vegetação natural do bioma cerrado, sendo adjacente a locais urbanos

De maneira geral, a região Centro-Oeste caracteriza-se predominantemente pelo clima quente, sendo sua característica 
mais marcante a frequência quase que diária de temperaturas altas. Nos meses mais quentes, setembro e outubro, podem ocorrer máximas superiores a $40^{\circ} \mathrm{C}$ (OLIVEIRA, 2007). Os tratamentos avaliados foram armadilhas plásticas do tipo ovitrampas de duas cores, preta e brancas, instaladas em duas alturas, no nível do solo e a $1 \mathrm{~m}$ de altura. As armadilhas continham água, e um graveto de madeira, que auxilia o mosquito a fazer a postura dos ovos.

$\mathrm{Na}$ área de estudo, foram instaladas armadilhas em 4 pontos, sendo o ponto 1 situado entre nas coordenadas latitude $15^{\circ} 36^{\prime} 44,6^{\prime}$ ' S e longitude $56^{\circ} 06^{\prime} 44,8^{\prime \prime} \mathrm{W}$, o ponto 2 latitude $15^{\circ} 36^{\prime} 45,6^{\prime \prime} \mathrm{S}$ e longitude $56^{\circ} 6^{\prime} 45,3^{\prime \prime} \mathrm{W}$, o ponto 3 latitude $15^{\circ} 36^{\prime} 46,3^{\prime \prime}$ S e longitude $56^{\circ} 06^{\prime}$ ' $46,1^{\prime \prime}$ W e o ponto 4 latitude $15^{\circ} 36^{\prime} 46,4$ " S e longitude $56^{\circ} 06^{\prime} 45,2$ " W (Figura 1).

Figura 1 - Área de estudo e dos pontos de coleta. Área de vegetação nativa, Município de Cuiabá, MT, 2017

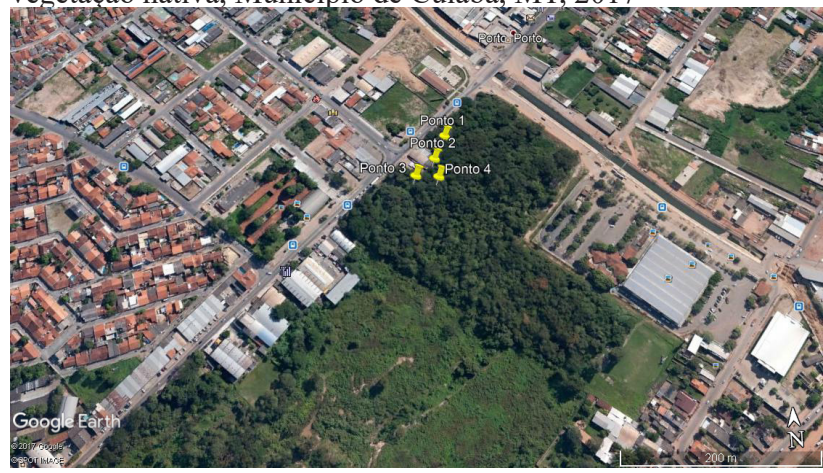

Fonte: Adaptado de Google Earth.

\subsection{Procedimentos de coleta e amostragem}

As populações dos mosquitos foram obtidas a partir de larvas e ovos coletados nas armadilhas ovitrampas instaladas na área de estudo. Em cada ponto foram instaladas armadilhas de cor preta e de cor branca, dois para cada cor, sendo duas armadilhas instaladas ao nível do solo e duas a um metro e meio acima do nível do solo, assim totalizando 16 armadilhas (Figura 2).

Figura 2 - Armadilhas ovitrampas instaladas na área de estudo. (A) Armadilha aérea de coloração preta; (B) Armadilha aérea de coloração branca; (C) Armadilha de solo na coloração escura; (D) Armadilha de solo na coloração preta. Área nativa, Município de Cuiabá, MT, 2017

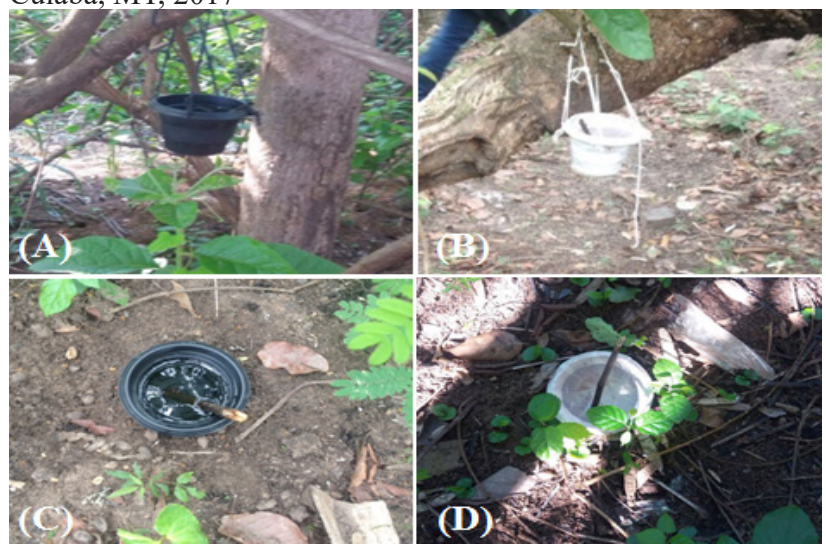

Fonte: Autores.
As coletas foram realizadas semanalmente entre o período de novembro a dezembro de 2016, sendo 6 coletas no total, assim em cada coleta as armadilhas foram preenchidas com água novamente. O material contido nas armadilhas, contendo larvas e ovos foi colocado em recipientes de plásticos devidamente identificados de acordo com o tratamento e local. Posteriormente, foram encaminhados para o laboratório de microbiologia ambiental da universidade de Cuiabá (UNIC) para contagem e identificação.

A água coletada das armadilhas foi depositada em recipientes oriundos de garrafas pet cortadas, com uma tela na abertura para impedir a saída dos adultos e permitir sua contagem e identificação (Figura 3).

Figura 3. Criação das larvas e pupas coletadas no experimento. Laboratório de Microbiologia ambiental, UNIC, Cuiabá 2016

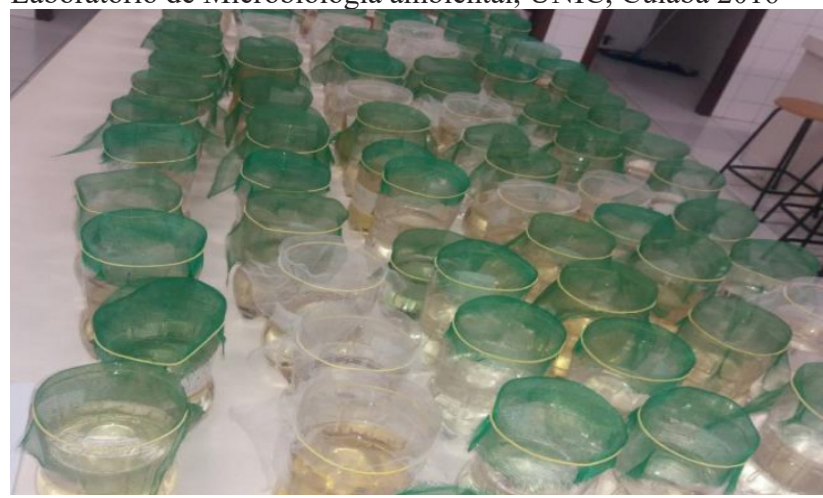

Fonte: Autores.

Os recipientes foram mantidos em ambiente escuro e climatizado com $23^{\circ} \mathrm{C} \pm 2$ escura. Cada recipiente recebeu ração para alevinos $1 \mathrm{~g}$ pra $500 \mathrm{~mL}$ de água, assim garantindo a sobrevivência da larva e emergência do adulto para posterior identificação.

As avaliações de laboratório foram realizadas três vezes por semana até o nascimento do ultimo mosquito. Para cada coleta foram realizadas seis avaliações, da qual foram quantificados o número de larvas, pupas e adultos. Entre os adultos a contagem foi feita com a identificação da espécie e separação por gênero. Após as avaliações os indivíduos foram mortos com álcool $70 \%$.

Para saber se existem diferenças entre as médias de indivíduos coletados nas armadilhas em função da posição de instalação, seguiu o experimento em delineamento inteiramente casualizado em esquema fatorial $2 \times 2$ (cor $\mathrm{x}$ posição), assim os dados foram submetidos a análise de variância e as médias observadas foram comparados pelo teste de Tukey ao nível de 5\% de probabilidade de erro. Para uniformização e normalidade dos dados, estes foram transformados utilizando a fórmula: $\sqrt{X+0,5}$, devido a se tratar de dados de contagem (BANZATTO; KRONKA, 2006). As análises estatísticas foram executadas por meio do software R (CORE TEAM, 2020). 


\subsection{Resultados e Discussão}

Ao todo foram coletados 357 mosquitos, em que houve maior ocorrência de mosquitos da espécie Ae. Albopictus, sendo coletados 278 indivíduos, 78\% do total (Quadro 1). A ocorrência de insetos fêmea foi maior para ambas as espécies, sendo 44 e 137 para Ae. Aegyptis e Ae albopictus respectivamente. Em relação à posição de instalação, percebeu-se que houve maior ocorrência de indivíduos em armadilhas aéreas, $56 \%$ do total.

Quadro 1 - Quantidades de mosquitos coletados em cada ponto amostrado ao longo do período analisado, de acordo com a espécie e sexo.

\begin{tabular}{|c|c|c|c|c|c|c|}
\hline \multirow{2}{*}{ Posição } & \multicolumn{2}{|c|}{ Aedes aegyptis } & \multicolumn{2}{|c|}{ Aedes albopíctus } & \multirow[t]{2}{*}{ Total } & \multirow{2}{*}{$\begin{array}{l}\text { Demais } \\
\text { espécie* }\end{array}$} \\
\hline & $\hat{0}$ & q & $\hat{o}$ & o & & \\
\hline Aérea & 29 & 27 & 71 & 73 & 200 & 245 \\
\hline Solo & 6 & 17 & 70 & 64 & 157 & 250 \\
\hline Total & 35 & 44 & 141 & 137 & 357 & 495 \\
\hline
\end{tabular}

* espécies que não pertencem ao gênero Aedes.

Legenda: (đ) Símbolo masculino, (ㅇ) símbolo feminino.

Fonte: Dados da pesquisa.

Conforme relataram O'meare et al. (1995) e Suwonkerd et al. (1996) em um estudo realizado na Flórida (EUA) e Tailândia, constataram que, quando essas espécies de Aedes coincidem nos criadouros, há uma tendência ao aumento de Ae. albopictus, tanto em áreas urbanas quanto em zonas rurais.

O Ae. albopictus opta depositar seus ovos em ocos de árvores, mesmo com facilidade de se adaptar ao ambiente antrópico (SILVA et al., 2009). De acordo com observações realizada por Lima-Camara et al. (2006), a modificação antrópica no ambiente e o aumento do grau de cobertura vegetal podem influenciar na distribuição e frequência do Ae. albopictus, ocasionado índices de infestação mais elevados em áreas suburbanas.

No estudo de segregamento de habitat, Branks et al. (2003) constataram que o habitat influenciou na abundância de $A e$. aegypti e de Ae. albopictus, com predomínio do Ae. aegypti em áreas de elevada concentração urbana e Ae. albopictus em áreas rurais. Tais autores verificaram abundância semelhante de ambas as espécies na maior parte das áreas suburbanas analisadas.

No estudo de Santos (2008), em Jaboatão dos Guararapes - PE, a existência das espécies Ae. aegypti e Ae. Albopictus foi observada em todos os bairros e aproximadamente $30 \%$ das ovitrampas houve a manifestação de ambas as espécies. Neste município, $A$. albopictus foi à espécie dominante em 2002, 55,4\% das 4.443 larvas identificadas, já em 2003, a maior frequência foi de $A$. aegypti com $65,3 \%$. Ainda segundo o autor, locais com cobertura vegetal demonstra que Ae. albopictus ocorre com maior frequência e abundância se comparados as áreas com cobertura vegetal escassa. O que corrobora com os resultados obtidos, visto que o estudo foi em ambiente natural e houve maior concentração de indivíduos da espécie Ae. Albopictus.

Em relação à sobrevivência das larvas no laboratório, as armadilhas aéreas de coloração preta, obtiveram o maior número de indivíduos em relação à coloração branca, pois $65 \%$ das larvas coletadas foram obtidas das armadilhas pretas (Quadro 2).

Quadro 2 - Número de larvas e pupas coletadas nas armadilhas de coloração preta e branca instaladas no solo e a 1,5 m acima do solo

\begin{tabular}{|c|c|c|c|c|c|c|c|c|}
\hline \multirow{3}{*}{ Leituras } & \multicolumn{4}{|c|}{ Aérea } & \multicolumn{4}{|c|}{ Solo } \\
\hline & \multicolumn{2}{|c|}{ Preta } & \multicolumn{2}{|c|}{ Branca } & \multicolumn{2}{|c|}{ Preta } & \multicolumn{2}{|c|}{ Branca } \\
\hline & Larva & Pupa & Larva & Pupa & Larva & Pupa & Larva & Pupa \\
\hline 1 & 84 & 27 & 10 & 02 & 127 & 37 & 73 & 31 \\
\hline 2 & 32 & 13 & 01 & 08 & 13 & 69 & 02 & 26 \\
\hline 3 & 16 & 31 & 01 & 02 & 10 & 35 & 13 & 04 \\
\hline 4 & 08 & 38 & $*$ & $*$ & $*$ & 25 & 01 & 10 \\
\hline 5 & 08 & 09 & $*$ & 01 & $*$ & 19 & $*$ & 10 \\
\hline 6 & $*$ & 08 & $*$ & $*$ & $*$ & 13 & $*$ & $*$ \\
\hline
\end{tabular}

Legenda: $(*)$ não foram observadas novas larvas e pupas.

Fonte: Dados da pesquisa.

Nas leituras 1 e 2 foram observou-se os maiores números de larvas e pupas e ainda, relação à sobrevivência em laboratório, é possível constatar que ao longo do tempo as larvas mudavam para a fase de pupa durante os intervalos de leitura, pois novas larvas iam eclodindo, a cada leitura, evidenciando assim que nas coletas em campo, além de larvas também tinham ovos.

No Quadro 3, observa-se o número de mosquitos de Ae. aegypti e Ae. albopictus coletados nas leituras semanais das armadilhas instaladas no solo e aérea, sendo possível perceber que decorridos 3 dias após a coleta, já pode ocorrer eclosão de adultos. O pico de maior concentração de eclosão ocorreu entre a segunda e quarta leitura, da qual demonstra que decorridos seis dias após a coleta, a maior parte dos indivíduos finalizaram o ciclo de desenvolvimento. 
Quadro 3 - Quantidade e identificação dos mosquitos adultos coletados nas armadilhas instaladas no solo e a $1 \mathrm{~m}$ acima do solo em função do tempo

\begin{tabular}{|c|c|c|c|c|c|c|c|c|}
\hline \multirow{3}{*}{ Leituras } & \multicolumn{4}{|c|}{ Aérea } & \multicolumn{4}{|c|}{ Solo } \\
\hline & \multicolumn{2}{|c|}{ Ae. aegypti } & \multicolumn{2}{|c|}{ Ae. albopictus } & \multicolumn{2}{|c|}{ Ae. aegypti } & \multicolumn{2}{|c|}{ Ae. albopictus } \\
\hline & $\hat{\sigma}$ & q & $\hat{\sigma}$ & q & $\hat{0}$ & q & $\hat{\sigma}$ & ㅇ \\
\hline 1 & 4 & 2 & 5 & 8 & $*$ & $*$ & 14 & 13 \\
\hline 2 & 9 & 8 & 21 & 10 & 3 & 5 & 21 & 27 \\
\hline 3 & 7 & 11 & 22 & 21 & 2 & 8 & 12 & 13 \\
\hline 4 & 5 & 5 & 11 & 15 & 1 & 4 & 18 & 8 \\
\hline 5 & 3 & 1 & 7 & 12 & $*$ & $*$ & 4 & 3 \\
\hline 6 & 1 & $*$ & 5 & 7 & $*$ & $*$ & 1 & $*$ \\
\hline Total & 29 & 27 & 71 & 73 & 6 & 17 & 70 & 64 \\
\hline
\end{tabular}

Legenda: * Não houve manifestação de mosquito.

Fonte: Dados da pesquisa.

De acordo com Mori (1979) em uma pesquisa realizada no Japão, observou-se que quando as larvas de Ae. albopictus são encontradas em baixa densidade, o período larval é menor e a taxa de manifestação dos adultos é maior, resultando em fêmeas grandes e com capacidade de pôr muitos ovos. Porém, essas fêmeas tendem a ficar adjuntas aos criadouros e a realizar pequenos repastos sanguíneos após a manifestação. Assim, a população de Ae. albopictus tende a sofrer um acréscimo gradativo. No entanto, quando as larvas são criadas em alta densidade, a tendência é inversa. Baseado nessas evidências constata-se que a população de larvas de Ae. albopictus é regulada primariamente pela própria densidade larval na área.

Os mosquitos da espécie Ae. aegypti tiveram maior número de manifestações nas armadilhas de colocação preta, tanto aérea quanto solo. Já em relação à diferença significativa, percebe que os mosquitos em chance de escolha de posição de armadilhas, optam pelas instaladas a $1,5 \mathrm{~m}$ acima do nível do solo, já em relação à coloração percebe se a forte influência da coloração preta, em que as fêmeas ovipositam seus ovos preferencialmente nessas armadilhas (Quadro 4).

Quadro 4 - Diferença estatística entre indivíduos adultos de Aedes aegypti coletados nas armadilhas de cor branca e preta em função da posição de instalação

\begin{tabular}{|l|r|l|r|l|}
\hline \multirow{2}{*}{ Cor } & \multicolumn{3}{|c|}{ Posição de instalação } \\
\cline { 2 - 5 } & \multicolumn{2}{|c|}{ Solo } & \multicolumn{2}{c|}{ Aérea } \\
\hline Branco & $1 \pm 0,95$ & bA & $0,5 \pm 0,57$ & bA \\
\hline Preto & $3 \pm 0,81$ & aB & $8 \pm 2,1$ & aA \\
\hline Significância & \multicolumn{3}{|c|}{$0,0011^{* *}$} \\
\hline CV (\%) & \multicolumn{3}{|c|}{17,37} \\
\hline
\end{tabular}

1 Cálculo efetuado com os dados transformados pela fórmula: $\sqrt{X+0,5}$

** Significativo a $1 \%$ de probabilidade de erro.

Médias seguidas de mesma letra minúsculas nas colunas e maiúsculas nas linhas não diferem estatisticamente entre si ao nível de $5 \%$ de probabilidade de erro pelo teste de Tukey.

Fonte: Dados da pesquisa.

Forattini e Brito (2003) apontaram que a existência de reservatórios domésticos, mesmo de coloração diferente da escura, pode prover condições favoráveis à manutenção de populações de $A e$. aegypti, até mesmo em períodos de baixas precipitações. Segundo Gomes et al. (2003) fêmeas de $A e$. aegypti optam em ovipositar seus ovos em recipientes de cores escuras, com bocas largas, sendo os ovos ovipositados em superfícies ásperas e úmidas e um pouco acima da linha d'água.

Serpa et al. (2006) averiguou que a maior abundância de Ae. aegypti em comparação ao Ae. albopictus em área urbana, inicialmente infestada por esta última espécie, mostrou maior habilidade do Ae. aegypti em propagar-se em pneus. A existência desse criadouro no ambiente pode ser importante na conservação e abundância do Ae. aegypti. No estudo em questão a área analisada não é uma área totalmente urbanizada, sendo uma área de vegetação nativa.

Em relação a Ae albopictus, houve diferença significativa entre a coloração das armadinhas. Observou-se maior ocorrência nas armadilhas de cor preta, tanto em posição aérea quanto no solo (Quadro 5).

Quadro 5 - Diferença estatística dos indivíduos adultos de Aedes albopictus coletados nas armadilhas de cor branca e preta em função da posição de instalação

\begin{tabular}{|l|l|l|l|l|}
\hline \multirow{2}{*}{ Cor } & \multicolumn{4}{|c|}{ Posição $^{1}$} \\
\cline { 2 - 5 } & \multicolumn{2}{|c|}{ Solo } & \multicolumn{2}{c|}{ Aérea } \\
\hline Branco & $11 \pm 1,5$ & bA & $3 \pm 1,2$ & bB \\
\hline Preto & $30 \pm 6,2$ & aA & $28 \pm 3,1$ & aA \\
\hline Significância & \multicolumn{4}{|c|}{$0,0001^{* *}$} \\
\hline CV (\%) & \multicolumn{4}{|c|}{18,92} \\
\hline
\end{tabular}

Cálculo efetuado com os dados transformados pela fórmula: $\sqrt{X+0,5}$ ** Significativo a $1 \%$ de probabilidade de erro. Médias seguidas de mesma letra minúsculas nas colunas e maiúsculas nas linhas não diferem estatisticamente entre si ao nível de $5 \%$ de probabilidade de erro pelo teste de Tukey.

Fonte: Dados da pesquisa.

Nas armadilhas brancas, as duas espécies preferiram significativamente as armadilhas posicionadas no solo. Possivelmente, por ficarem sombreadas, assim apresentando uma coloração mais escura se comparada com as instaladas na parte aérea. Consoli e Oliveira (1994) destacaram que a fêmea desse inseto é atraída por recipientes escuros e sombreada, com superfície áspera propícia para o pouso.

A ocorrência de distribuição Ae. aegypti em área nativa, justifica-se uma vez que muitos fatores ambientais, propiciam a proliferação desta espécie, pois alguns locais exibem mais criadouros do que outros, por exemplo, a presença de 
vegetação nativa ou de materiais descartados em quintais e terrenos baldios que se tornam pontos ideais para a oviposição. Aliás, esta espécie também pode exibir tendência genética a se agrupar e provocar a distribuição contagiosa, independentemente dos fatores ambientais (ALMEIDA et al., 2013).

Como resultado da intensificação da urbanização em áreas verde cada vez maior, Ae. albopictus gradativamente vem ocorrendo no ambiente urbano das grandes cidades ao lado do Ae. aegypti. Apesar do predomínio deste culicídeo em áreas urbanas intensamente povoadas (CHRISTOPHERS, 1960), larvas de Ae. albopictus têm sido frequentemente encontradas evoluindo-se nos mesmos criadouros que as larvas de $A e$. aegypti (BRAKS et al., 2003). Nestes criadouros, as taxas de desenvolvimento das duas espécies sofrem alterações, de acordo com o número de indivíduos e com o tipo e a oferta de alimento (BARRERA, 1996).

\section{Conclusão}

A armadilha ovitampa de coloração preta instalada a 1,5m do solo foi mais eficiente para a contagem de mosquitos da espécie Ae. aegypti.

Para a espécie Ae. albopictus a armadilha de coloração preta foi mais eficiente para a contagem de indivíduos independente da altura instalada, ao nível do solo ou a 1,5m.

\section{Referências}

ALMEIDA, C.O.S.; AMORIM, R.S.S.; COUTO, E.G. Potencial erosivo da chuva de Cuiabá, MT: distribuição e correlação com a precipitação pluviométrica. Rev Bras. Eng. Agríc. Amb., v.15, n.2, p.178-184, 2011. doi: 10.1590/S1415-43662011000200011

ALMEIDA, P.S. et al. Infestação de Aedes aegypti (Linnaeus, 1762) (Diptera: Culicidae) determinada por armadilhas de oviposição (ovitrampas) no município de Costa Rica, Estado de Mato Grosso do Sul. Rev. Patol. Trop.. v.42, n.3, p.331-339, 2013. doi: $10.5216 /$ rpt.v42i3.26929

ARAÚJO, W. S. Aspectos legais e conceituais de estudos epidemiológicos aplicados à avaliação de potencial malarígeno no Brasil. Physis, v.29, n.3, p1-3, 2019. doi: 10.1590/S010373312019290302

BANZATTO, D. A.; KRONKA, S. N. Experimentação Agrícola. Jaboticabal: Funep, 2006.

BARATA, E.A.M.F. et al. População de Aedes aegypti (L.) em área endêmica de dengue, Sudeste do Brasil. Rev. Saúde Pública, v.35, n.3, p.237-242, 2001. doi: 10.1590/S0034-89102001000300004.

BARRERA R. Competition and resistance to starvation in larvae of container-inhabiting Aedes mosquitoes. Ecol. Entomol., v.21, n.2, p.117-127, 1996. doi: 10.1111/j.1365-2311.1996.tb01178.x

BRAGA, A.I., VALLE, D. Aedes aegypti: inseticidas, mecanismos de ação e resistência. Epidemiol. Serv. Saúde, v.16, n.4, p.279293, 2007. doi: 10.5123/S1679-49742007000400006

BRAKS, M. A. et al. Convergent habitat segregation of Aedes aegypti and Aedes albopictus (Diptera: Culicidae) in Southeastern Brazil and Florida. J. Med. Entomol., v.40, n.6, p.785-794, 2003. doi: 10.1603/0022-2585-40.6.785

BRASIL. Ministério da Saúde. Fundação Nacional de Saúde. Instruções para pessoal de combate ao vetor - manual de normas técnicas. Brasília, 2001.

CASTRO, M. G; et al. Dengue virus detection by using reverse transcription-polymerase chain reaction in saliva and progeny of experimentally infected Aedes albopictus from Brazil. Memórias do Instituto Oswaldo Cruz. v.99, n.8, p.809-814. 2004. doi: 10.1590/S0074-02762004000800005

CHRISTOPHERS, S. R. Aedes aegypti (L.) the yellow fever mosquito. Its life history bionomics and structure. London: Cambridge University Press. 1960, 739p.

CONSOLI, R. A. G. B.; OLIVEIRA, R. L. Principais mosquitos de importância sanitária no Brasil. Rio de Janeiro: Fiocruz, 1994, 228p.

CORE TEAM. R: Uma linguagem e ambiente para computação estatística. $R$ Fundação para Computação Estatística, Viena. Disponível em; http://www.R-project.org. Acesso em 19 set. 2020.

COSTA, I. P; NATAL, D. Distribuição Espacial da Dengue e Determinante Socioeconômico em Localidade Urbana no Sudeste do Brasil. Rev. Saúde Pública, v.32, n.3 p. 232-236, 1998. doi: 10.1590/S0034-89101998000300005

DEGALLIER, N. et al. Aedes albopictus may not be vector of dengue virus in human epidemics in Brazil. Revista Saúde Pública. v.37, n.3, p.386-393. 2003. doi: 10.1590/S003489102003000300019

DONALÍSIO, M.R.; GLASSER, C.M. Vigilância entomológica e controle de vetores do dengue. Rev. Bras. Epidemiol., v.5 n3. p.259-272. 2002. doi: 10.1590/S1415-790X2002000300005

FORATTINI, O. P.; BRITO, M. Reservatórios domiciliares de água e controle do Aedes aegypti. Rev. Saúde Pública, v.37, n.5, p.676-677, 2003. doi: 10.1590/S0034-89102003000500021

FORATTINI, P. P. Culicidologia médica: identificação, biologia, epidemiologia. São Paulo, Edusp, 2002.

GOMES, A. C. et al. Host-feeding patterns of potential human disease vectors in the Paraíba Valley Region, State of São Paulo, Brazil. J. Vector Ecol., v.28, n.1, p.7478, 2003.

LIMA-CAMARA, T. N. et al. Frequência e distribuição espacial de Aedes aegypti e Aedes albopictus (Díptera, Culicidade) no Rio de Janeiro, Brasil. Cad. Saúde Pública, v.22, n.10, p.2079-2084, 2006. doi: 10.1590/S0102-311X2006001000013

LUCIANO-ALMEIDA, M.H.A.; KOETZ, M. Dengue: doença proveniente de problemas ambientais: o caso do Jardim dos Pioneiros, no Município de Rondonópolis - MT. Rev. Educ. Amb. Ação. n.37, v.10, s.3, 2011. Disponível em: < ttp://www.revistaea. org/artigo.php?idartigo=1114>. Acesso em: 30 mar. 2020.

MILlER, B. R.; BALLINGER, M. E. Aedes albopictus mosquitoes introduced into Brazil: vector competence for yellow fever and dengue viruses. Trans. Royal Soc. Trop. Med. Hyg., v.82, n.3, p.476-477, 1988. doi: 10.1590/0037-8682-0155-2014

MORI A. Effects of larval density and nutrition on some attributes of immature and adult Aedes albopictus. Trop. Med., v.21, p.85103, 1979.

O'MEARA, G.F. et al. Spread of Aedes albopictus and decline of Ae aegypti (Diptera: Culicidae) in Florida. J. Med. Entomol., v.32, n.4, p.554-562, 1995. doi: 10.1093/jmedent/32.4.554

OLIVEIRA, A. S. Análise de desempenho térmico e consumo de energia em residências na cidade de Cuiabá/MT: estudo de caso. 2007. Cuiabá: Universidade Federal de Mato Grosso, 2007.

OLIVEIRA, R.L. et al. Large genetic differentiation and low variation in vector competence for dengue and yellow fever viruses of Aedes albopictus from Brazil, the United States, and 
the Cayman Islands. Am. J. Trop. Med. Hyg., v.69, n.1, p.105-114, 2003. doi: 10.4269/ajtmh.2003.69.105

OLIVEIRA, S.E.T. Condições microclimáticas e a proliferação de vetores da dengue e febre amarela em uma escola de CuiabáMT. Cuiabá: Universidade Federal de Mato Grosso, 2013.

SANTOS, M. A. V. M. Aedes aegypti: estudos populacionais e estratégias integradas para controle vetorial em municípios da região metropolitana do Recife, no período de 2001 a 2007. Recife: Fundação Oswaldo Cruz, 2008.

SERPA, L. L. N. et al. Variação sazonal de Aedes aegypti e Aedes albopictus no município de Potim, São Paulo. Rev. Saúde Pública, v.40, n.6, p.1101-1105, 2006. doi: 10.1590/S003489102006005000008 .

SILVA, G. I. et al. Estudo do comportamento de oviposição no campo e o ciclo de vida de Aedes albopictus (SKUSE, 1894) (Diptera: Culicidae) em laboratório. Rev. Saúde Pública, v.38, n.1, p.45-51, 2009. doi: 10.5216/rpt.v38i1.6220

SILVA, S. J. et al. A dengue no brasil e as políticas de combate ao Aedes aegypti: da tentativa de erradicação ás políticas de controle.
Hygeia Rev. Bras. Geog. Méd. Saúde. v.3, n.6, p.163-175, 2008.

SUWONKERD, W. et al. Seasonal occurrence of Ae. albopictus in used tires in 1992- 1994, Chiangmai, Thailand. Trop. Med., v.38, n.3, p101-105, 1996. doi: 10.7601/mez.48.22_2

SOUZA, E. Estratégias de uso de AedesTrap para o monitoramento de Aedes (stegomyia) aegypti (L.) (Diptera: Culicidae). Recife: Universidade Federal de Pernambuco, 2010.

TAUIL, P. L. Aspectos críticos do controle do dengue no Brasil. Cadernos de Saúde Pública, v.18, n.3, p.867-871, 2002. doi: 10.1590/S0102-311X2002000300030.

TILAK, R. et al. A laboratory investigation into oviposition responses of Aedes aegypti to some common household substance sandwa terfrom conspecific larvae. Med. J. Armed Forces India, v.61, n.3, p.227-229, 2004. doi: 10.1016/S0377-1237(05) 801595

ZARA, A.S.L.A. et al. Estratégias de controle do Aedes aegypti: uma revisão. Epidemiol. Serv. Saúde, v.25, n.2, p.391-404, 2016. doi: 10.5123/s1679-49742016000200017. 